\title{
A Noncooperative Power Control Game for Multirate CDMA Data Networks
}

\author{
Chi Wan Sung, Member, IEEE, and Wing Shing Wong, Fellow, IEEE
}

\begin{abstract}
The authors consider a multirate code-division multiple acess system, in which all users have the same chip rate and vary their data rate by adjusting the processing gain. The receivers are assumed to be implemented using conventional matched filters, whose performance is sensitive to the received power levels. The authors' goal is to maximize the total system throughput by means of power control. A game theoretic approach is adopted. It is shown that for a certain type of pricing function, a unique Nash equilibrium solution exists and it possesses nice global properties. For example, it can be shown that for the optimal solution a high-rate connection should maintain a higher energy per bit than low-rate ones. The asymptotic spectral efficiency is also derived.
\end{abstract}

Index Terms-Multirate CDMA systems, Nash equilibrium, noncooperative game, power control.

\section{INTRODUCTION}

$\mathbf{S}$ PEECH provisioning, a low-data rate service, is the major objective of second-generation cellular systems. However, as the demand for wireless services proliferates, future wireless systems should be able to accommodate more diverse service types. Inherently, voice, data, and video services require different data rates. The bit rate requirement may range from a few kilobytes per second to as much as $2 \mathrm{Mb} / \mathrm{s}$. For this reason, it is necessary to support multirate transmission in third-generation wireless networks.

In this paper, we consider the problem of providing multirate transmission using direct-sequence code-division multiple access (DS/CDMA). There are different ways to design a multirate CDMA system. The one to be examined here is called variable spreading gain access. In this method, the signals of all users are spread to the same bandwidth by keeping the chip rate identical. As a consequence, different data rates result in different spreading gains. In such a scenario, the traditional method of keeping the received power constant is inappropriate. Intuitively, a solution in which the energy per bit for all users is constant seems optimal. Indeed, we will show that in some asymptotic situations such a solution maximizes the

Manuscript received December 1, 1999; revised November 1, 2000; accepted July 12,2002 . The editor coordinating the review of this paper and approving it for publication is S. Tekinay. This work was supported in part by a Grant from City University of Hong Kong under Project 7001234 and in part by a Grant from the Research Grants Council of the Hong Kong Special Administrative Region under Project CUHK4222/00E.

C. W. Sung is with the Department of Computer Engineering and Information Technology, City University of Hong Kong, Kowloon, Hong Kong (e-mail: itcwsung@cityu.edu.hk).

W. S. Wong is with the Department of Information Engineering, The Chinese University of Hong Kong, Shatin, N.T., Hong Kong (e-mail: wswong@ie.cuhk.edu.hk).

Digital Object Identifier 10.1109/TWC.2002.806394 system throughput. However, to achieve optimality in general, a high-rate connection should maintain a higher energy per bit than low-rate connections.

In the literature, power control studies tend to focus on devising algorithms to provide acceptable quality for all the connections (see, e.g., [5], [10], [11], [16], [17]). This is usually translated into a minimum requirement on the signal-to-interference ratio (SIR). This formulation is suitable for voice traffic since there is dubious, if any, gain in user satisfaction to have improvement of the SIR beyond an acceptable threshold, commonly called the target SIR. For data traffic, however, the situation is different. Typically, a data packet in error needs to retransmit. A higher SIR reduces the number of retransmissions, thus minimizing the delay while maximizing effective throughput. So there is no natural way to determine a target SIR. In view of this, we consider a more relevant formulation, in which the total throughput is to be maximized. Some related studies can be found in [7] and [12].

Recently, a game theoretic approach to the power control problem for data traffic has been offered in [2], [4], [8], [9], and [13]. The distributed power control problem is formulated as a noncooperative game. The fundamental difference between our work and previous works lies in the definition of the payoff function. In [8] and [9], the payoff is defined through the bit error rate of a noncoherent frequency-shift keying (FSK) scheme. A drawback of this definition is that the payoff goes to infinity when a user transmits at zero power. This degenerate situation arises from the fact that one-half of the bits can still be received correctly even if one transmits nothing at all. To remedy this problem, the definition was modified in an ad hoc way in [8] and [9]. In this paper, we overcome this problem by using an information theoretic approach. We define the payoff as the reliable information rate through the channel [1]. When the transmit power goes to zero, the information rate through the channel also diminishes to zero. So, the degenerate case does not occur in our formulation. As a consequence of this change in the definition of the payoff function, one can obtain a generalization of the asymptotic result on the spectral efficiency of CDMA systems.

The rest of the paper is organized as follows. In Section II, the system model is described. In Section III, we introduce the game theoretic framework and define our game model. In Section IV, a pricing mechanism is described. In Section V, it will be shown that with pricing, the resulting Nash equilibrium has a nice global property. In Section VI, we will derive the resulting spectral efficiency, which is defined as the maximum number of bits per chip that can be transmitted reliably through the channel [14]. The spectral efficiency of a single-rate 
CDMA system is derived in [3]. We show that a multirate system can achieve the same efficiency. In Section VII, the numerical results are presented. In Section VIII, conclusions are summarized.

\section{SYSTEM MODEL FOR MUlTIRATE CDMA SySTEMS}

We consider a single-cell CDMA system, in which there are $N$ active terminals. Terminal $i$ transmits its signal at rate $R_{i}$. All users have the same chip rate $R_{c}$, thus spreading their signal to the same bandwidth $W=R_{c}$. We assume that $R_{c}$ is an integral multiple of $R_{i}$ and the processing gain of user $i$ is defined by $L_{i}=R_{c} / R_{i} \geq 1$. Let $P_{i}$ be the transmit power of terminal $i$. The received power at the base station is $Q_{i}=G_{i} P_{i}$, where $G_{i}$ is the attenuation factor.

Though our results can be applied to chip-asynchronous systems, we assume that the system is chip-synchronous for simplicity. Let $a_{i}= \pm 1$ be the data symbol of user $i$ and $y_{i}$ be the corresponding decision variable at the receiver output. When matched filter is used, the channel can be modeled by the following conditional probability ${ }^{1}$ :

$$
P\left(y_{i} \mid a_{i}=w\right)=\frac{1}{\sqrt{2 \pi}} \exp \left(-\left(y_{i}-w \sqrt{2 E_{b, i} / J_{0, i}}\right) / 2\right)
$$

where $E_{b, i}=Q_{i} L_{i} T_{c}$ is the bit energy of user $i$, and $J_{0, i}$ is the interference spectral density defined by ${ }^{2}$

$$
\frac{J_{0, i}}{2}=\frac{T_{c}}{2} \sum_{j \neq i} Q_{j}+\frac{N_{0}}{2} .
$$

This channel is called a binary-input Gaussian-output (BIGO) channel. Its capacity is given by [15]

$$
f_{\mathrm{BIGO}}(x)=-\frac{1}{2} \log _{2} 2 \pi e-\int_{-\infty}^{\infty} P(y) \log _{2} P(y) d y
$$

where

$$
P(y)=\frac{P_{0}(y)+P_{0}(-y)}{2}
$$

and

$$
P_{0}(y)=\frac{1}{\sqrt{2 \pi}} \exp \left[-(y-\sqrt{2 x})^{2} / 2\right] .
$$

If the output of the BIGO channel is hard quantized into two levels, then the channel becomes a binary symmetric channel (BSC) with crossover probability

$$
p(x)=\frac{1}{2} \operatorname{erfc}(\sqrt{x})
$$

where

$$
\operatorname{erfc}(x)=\frac{2}{\sqrt{\pi}} \int_{x}^{\infty} e^{-t^{2}} d t
$$

The channel capacity of a BSC is [1]

$$
f_{\mathrm{BSC}}(x)=1+p(x) \log _{2} p(x)+(1-p(x)) \log _{2}(1-p(x)) .
$$

${ }^{1}$ Here, we have invoked the Gaussian assumption that $y_{i}$ is Gaussian distributed.

${ }^{2}$ If the signals are not chip synchronized, $J_{0, i} / 2$, which represents the variance of $y_{i}$, should be defined as $\left(T_{c} / 3\right) \sum_{j \neq i} Q_{j}+\left(N_{0} / 2\right)[6]$.
As a notational convenience, we define $x_{i}=E_{b, i} / J_{0, i}$. Note that $x_{i}$ also equals $\Gamma_{i} L_{i}$ where

$$
\Gamma_{i}=\frac{Q_{i}}{\sum_{j \neq i} Q_{j}+N_{0} W}
$$

Denote the interference power experienced by user $i$ by $I_{i}$, that is,

$$
I_{i}=\sum_{j \neq i} Q_{j}+N_{0} W
$$

It follows that

$$
x_{i}=\frac{Q_{i} L_{i}}{I_{i}} .
$$

We define $f\left(x_{i}\right)$ as the rate in bits per channel use at which information can be reliably sent through the channel [1]. In general, it is an increasing function of $x_{i}$, while its explicit form depends on the modulation and coding scheme. Since user $i$ accesses the channel $R_{i}$ times per second, the corresponding information rate in bit per second is given by

$$
R_{i} f\left(x_{i}\right)
$$

We call the above term the throughput of user $i$. The total throughput of the system, $C_{T}$, is given by

$$
C_{T}=\sum_{i=1}^{N} R_{i} f\left(x_{i}\right) .
$$

By Shannon's channel capacity theorem, $f$ is upper bounded by $f_{\mathrm{BIGO}}$ or $f_{\mathrm{BSC}}$, depending on whether the channel output is hard quantized. In this work, we do not stick to a specific modulation and coding scheme. We assume that $f$ is either $f_{\mathrm{BIGO}}$ or $f_{\mathrm{BSC}}$. It can be shown that for these two forms of $f$, the following conditions are satisfied (see Figs. 1 and 2 for a graphical illustration).

1) $f^{\prime}(x)>0 \forall x$. In particular, we have

$$
\lim _{x \rightarrow 0} f^{\prime}(x)= \begin{cases}\log _{2} e, & \text { for BIGO } \\ \frac{2}{\pi} \log _{2} e, & \text { for BSC. }\end{cases}
$$

2) $f^{\prime \prime}(x)<0 \forall x$.

3) $f^{\prime}(x)=o\left(x^{-2}\right)(x \rightarrow \infty)$.

4) Let $q(x)=2 f^{\prime}(x)+x f^{\prime \prime}(x)$. There exists $x_{0}$ such that

$$
q(x) \begin{cases}>0, & \text { for } 0 \leq x<x_{0} \\ =0, & \text { for } x=x_{0} \\ <0, & \text { for } x>x_{0}\end{cases}
$$

From Properties 1,2 , and 4 , it is easy to see that

$$
2 f^{\prime}(x)+(x+c) f^{\prime \prime}(x)<0 \quad \forall x
$$

for some constant $c$ that is large enough. Numerically, we find that the above statement is true for BIGO if $c>1.1$, and is true for BSC if $c>2.2$.

Though we consider these two forms of $f$ only, our results can be applied to other situations, provided that $f$ satisfies Properties 1-4. 

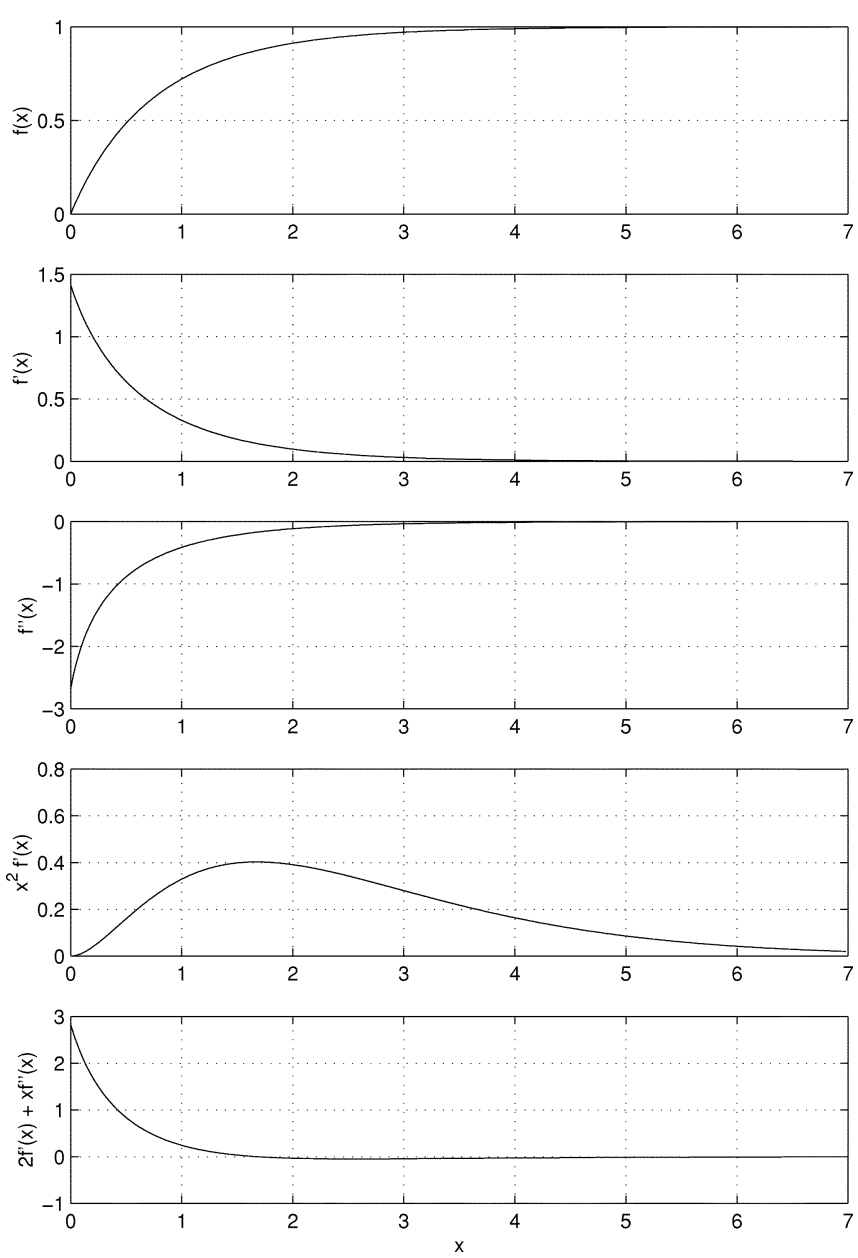

Fig. 1. $f_{\mathrm{BIGO}}(x)$ and its derivatives.

\section{POWER CONTROL GAME}

Generally speaking, our objective is to find a power vector which maximizes the total throughput. For practical reasons, it is most desirable if this can be achieved by letting each user adjust its power level, based on local information. This distributed operation fits well in a game theoretic framework proposed in [8] and [9]. We will first describe this framework. After introducing the terminology, we will construct a new game by defining the payoff function pertaining to our problem.

In a power control game, each mobile user is regarded as a player of the game. The strategy space of player $i$ is the interval $\mathcal{P}_{i}=\left[0, M_{i}\right]$. In practice, the power levels have finite ceilings. For theoretical study, sometimes we consider the case there is no upper limit on the power levels. In that case, $M_{i} \mathrm{~s}$ are equal to infinity. The joint strategy space $\mathcal{P}=\mathcal{P}_{1} \times \mathcal{P}_{2} \times \cdots \times \mathcal{P}_{N}$ is the Cartesian product of all the individual strategy spaces. Each player chooses a power level $P_{i} \in \mathcal{P}_{i}$. The payoff function of player $i$ is denoted by $u_{i}(\mathbf{P})$. Occasionally, an alternative notation $u_{i}\left(P_{i}, \mathbf{P}_{-i}\right)$ is used, where $\mathbf{P}_{-i}$ denotes the power vector of all users except user $i$.

The power control game (PCG) can be formally expressed as

$$
\max _{P_{i} \in \mathcal{P}_{i}} u_{i}\left(P_{i}, \mathbf{P}_{-i}\right) \quad \forall i=1,2, \ldots, N .
$$

In a PCG, each user chooses an appropriate power to maximize his payoff. As there is no cooperation among the users, it is im-
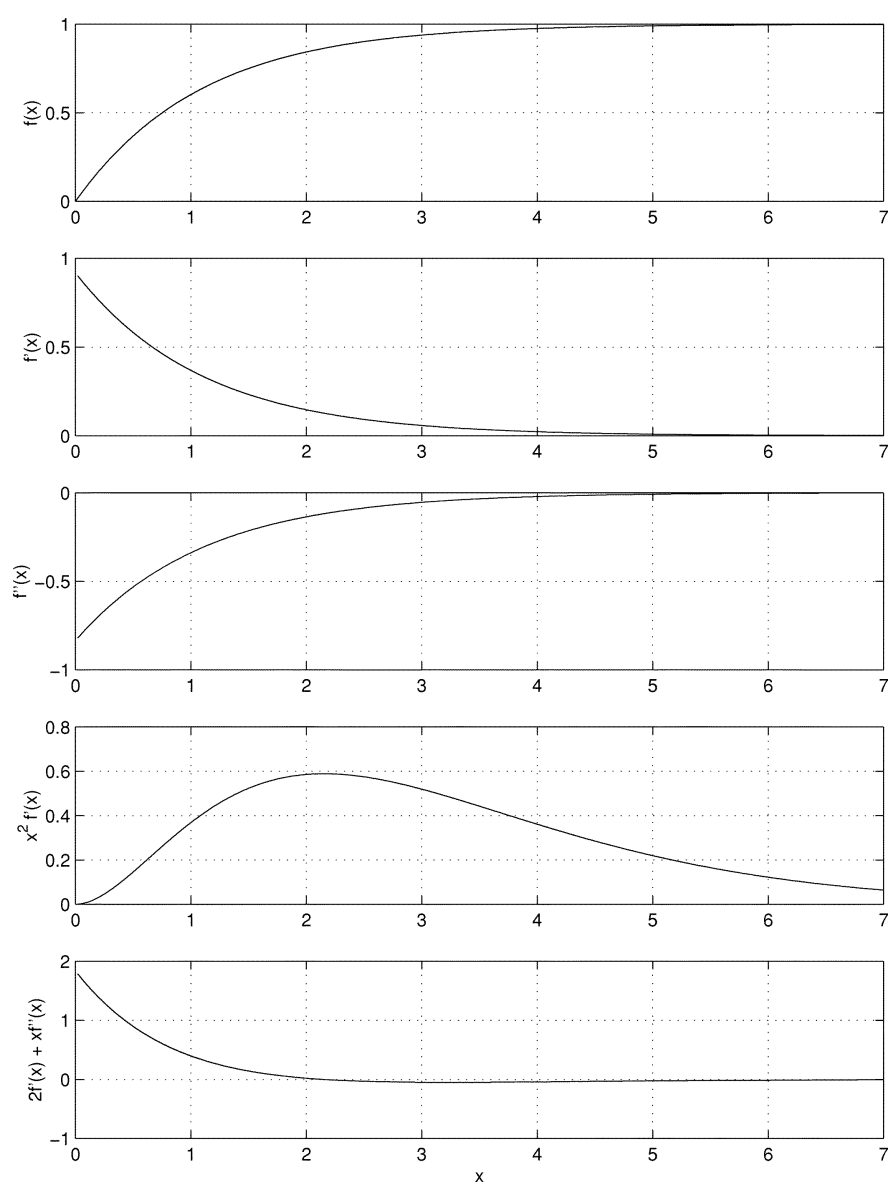

Fig. 2. $f_{\mathrm{BSC}}(x)$ and its derivatives.

portant to ensure the dynamic stability of the system. A concept which relates to this issue is the so-called Nash equilibrium.

Definition 1: A power vector $\mathbf{P}^{*}$ is a Nash equilibrium if, for every user $i$

$$
u_{i}\left(P_{i}^{*}, \mathbf{P}_{-i}^{*}\right) \geq u_{i}\left(P_{i}, \mathbf{P}_{-i}^{*}\right) \quad \forall P_{i} \in \mathcal{P}_{i} .
$$

A Nash equilibrium can be regarded as a stable solution, at which none of the users has the incentive to change its power. Many games have several Nash equilibria. To compare the qualities of two different solutions, a commonly used concept is called Pareto dominance.

Definition 2: A power vector $\mathbf{P}$ Pareto dominates another vector $\mathbf{P}^{\prime}$ if, for all $i$

$$
u_{i}(\mathbf{P}) \geq u_{i}\left(\mathbf{P}^{\prime}\right)
$$

and for some $j$

$$
u_{j}(\mathbf{P})>u_{j}\left(\mathbf{P}^{\prime}\right)
$$

Furthermore, a power vector $\mathbf{P}^{*}$ is Pareto optimal if there exists no vector which Pareto dominates $\mathbf{P}^{*}$.

The above framework is established in [8] and [9]. A PCG is completely defined once the payoff function is specified. In this paper, we consider the game where the payoff of user $i$ is its throughput, that is

$$
u_{i}=R_{i} f\left(x_{i}\right) .
$$


To distinguish this game from that in [8] and [9], we call it throughput maximization game (TMG). Note that the payoff of user $i$ depends only on $x_{i}$, which is completely determined by the received power vector, $\mathbf{Q}$. Without loss of generality, we treat $Q_{i}$ s as our independent variables. The strategy space of user $i$ becomes $\mathcal{Q}_{i}=\left[0, \tilde{M}_{i}\right]$ where $\tilde{M}_{i}=G_{i} M_{i}$, and the joint strategy space is $\mathcal{Q}=\mathcal{Q}_{1} \times \cdots \times \mathcal{Q}_{N}$.

Note that $Q_{i}$ can be written as a function of $\mathbf{x}=$ $\left[x_{1}, x_{2} \ldots, x_{N}\right]$

$$
Q_{i}(\mathbf{x})=\frac{x_{i}}{x_{i}+L_{i}} \times \frac{N_{0} W}{1-\sum_{j} \frac{x_{j}}{x_{j}+L_{j}}} .
$$

Furthermore, $Q_{i}\left(\mathbf{x}^{\prime}\right)>Q_{i}(\mathbf{x})$ if $\mathbf{x}^{\prime}>\mathbf{x}$. If there is no upper limit on the power levels (i.e., $\tilde{M}_{i}=\infty \forall i$ ), then given a positive vector, $\mathbf{x}$, a necessary and sufficient condition for $\mathbf{Q} \in \mathcal{Q}$ is

$$
\sum_{i=1}^{N} \frac{x_{i}}{x_{i}+L_{i}}<1 .
$$

We call this the feasibility condition [7]. If this condition holds, there is a one-one mapping between nonnegative $\mathbf{Q}$ and $\mathbf{x}$. Furthermore, the following equation always holds:

$$
\sum_{i} \frac{x_{i}}{x_{i}+L_{i}}=\frac{Q_{T}}{Q_{T}+N_{0} W}
$$

where $Q_{T}=\sum_{i} Q_{i}$.

The following theorem states that a Pareto optimal solution must be located at the boundary of the strategy space for a power control game with bounded strategy space.

Theorem 1 (Pareto Optimality): For a TMG with finite upper power ceilings for all users, a power vector $\mathbf{Q}$ is Pareto optimal if and only if $Q_{i}=\tilde{M}_{i}$ for some $i$.

Proof: Note that $u_{i}$ is a strictly increasing function of $x_{i}$. If $Q_{i}<\tilde{M}_{i}$ for all $i$, one can scale up $\mathbf{Q}$. The resultant $x_{i}$ s will all increase, thus improving the payoff of all players. Hence, $\mathbf{Q}$ cannot be Pareto optimal.

Now consider a vector $\mathbf{Q}$ with $Q_{i}=\tilde{M}_{i}$ for some $i$. If $\mathbf{Q}$ is not Pareto optimal, we can find another vector $\mathbf{Q}^{\prime}$ which Pareto dominates $\mathbf{Q}$. It implies that $x_{i}^{\prime} \geq x_{i}$ for all $i$, and $x_{j}^{\prime}>x_{j}$ for some $j$. Since $Q_{i}=x_{i} I_{i} / L_{i}$, it follows that

$$
\frac{Q_{j}^{\prime}}{I_{j}^{\prime}}>\frac{Q_{j}}{I_{j}}
$$

By adding 1 to both sides and simplifying

$$
Q_{j}^{\prime}>Q_{j}
$$

It then follows that $Q_{i}^{\prime}>Q_{i}$ for all $i$, which leads to a contradiction.

Since $u_{i}$ is a strictly increasing function of $Q_{i}$, for any given $\mathbf{Q}_{-i}$. It is easy to see that the TMG has an unique Nash equilibrium, which is achieved by setting the power of each user to its maximum value. By Theorem 1, this Nash equilibrium is Pareto optimal. In spite of this, this maximum power strategy may not be a good strategy from a global viewpoint. For instance, consider a user who is closest to the base station and requires only the lowest data rate. For the sake of other users, it is intuitively obvious that this user should not transmit at maximum power. Therefore, this strategy, though Pareto optimal, is not efficient from the system viewpoint. We consider a way to improve the system performance in the next section.

\section{PRicing Mechanism}

To find other strategies which improve the payoffs in a global sense, we use the method of pricing. This pricing mechanism can implicitly bring cooperation to the users, yet maintaining the noncooperative nature of the game. We let $c_{i}(\mathbf{Q})$ be the pricing function of player $i$. The discounted payoff function is defined as

$$
v_{i}(\mathbf{Q})=u_{i}(\mathbf{Q})-c_{i}(\mathbf{Q}) .
$$

The following is called a power control game with pricing (PCGP) [9]:

$$
\max _{Q_{i} \in \mathcal{Q}_{i}} v_{i}\left(Q_{i}, \mathbf{Q}_{-i}\right) \quad \forall i=1,2, \ldots, N .
$$

PCGP is essentially the same as PCG, except with a different payoff function. To distinguish between $u_{i}$ and $v_{i}$, from now on, we call $u_{i}$ the payoff and $v_{i}$ the discounted payoff of player $i$.

This pricing methodology is first applied to PCG in [9]. However, it is worth noting that our purpose of using pricing is different from that in [9]. Due to different definitions of the payoff function, the PCG considered in [9] possesses a Nash equilibrium which is not Pareto optimal. Thus they use pricing to bring about a Pareto improvement. In the TMG, the Nash equilibrium is Pareto optimal. Our intention of using pricing is to shape the users' behavior so as to improve the performance from a system viewpoint.

When a user transmits his information through the network, it causes interference to other users. To discourage this behavior, it is reasonable to charge the user some price for creating the interference. Intuitively, the pricing function of player $i$ should be a monotonic increasing function of his received power. Based on this argument, a linear pricing scheme is adopted in [8] and [9]. However, we suggest to normalize the received power by the total received power plus noise at the base station, that is

$$
c_{i}(\mathbf{Q})=\frac{\lambda Q_{i}}{\sum_{j=1}^{N} Q_{j}+N_{0} W}
$$

where $\lambda$ is the pricing parameter. The rationale of normalizing the received power is that the harm caused by player $i$ is based not only on the received power of player $i$, but also on the total interference at the base station. For example, consider the case $Q_{i}=10$. If $\sum_{j=1}^{N} Q_{j}+N_{0} W=20$, a large portion of the total interference is generated by user $i$. However, consider another scenario where $\sum_{j=1}^{N} Q_{j}+N_{0} W=200$. In this case, the harmful effect caused by player $i$ is comparatively small. Thus, the impact made by player $i$ is more accurately measured by the normalized received power.

With this pricing function, the discounted payoff function becomes

$$
v_{i}=R_{i} f\left(x_{i}\right)-\frac{\lambda Q_{i}}{Q_{i}+I_{i}} .
$$


Recall that $I_{i}$ is the interference experienced by user $i$. We call this game the throughput maximization game with pricing (TMGP).

For the pricing mechanism to be effective, $\lambda$ should not be too large or too small. To see this, we differentiate (26) with respect to $Q_{i}$

$$
\frac{\partial v_{i}}{\partial Q_{i}}=f^{\prime}\left(x_{i}\right) \frac{W}{I_{i}}-\frac{\lambda I_{i}}{\left(Q_{i}+I_{i}\right)^{2}} .
$$

If $\lambda>\left(\left(\tilde{M}_{i} / I_{i}\right)+1\right)^{2} f^{\prime}(0) W$, then $\partial v_{i} / \partial Q_{i}$ is always less than zero. Thus the optimal solution is obtained by setting all $Q_{i}$ s to zero. On the other extreme, if $\lambda=0$, it reduces to the original TMG. The following theorem describes some properties of the TMGP for a certain range of values of $\lambda$.

Theorem 2 (Existence and Uniqueness of Nash Equilibrium): Consider a power control problem with finite upper power bounds for all users. If the processing gain, $L_{i}$, of each user is greater than two for the BIGO case, and is greater than three for the BSC case, then there exists an unique $\lambda^{*}$ such that for any $\lambda \in\left[\lambda^{*}, W f^{\prime}(0)\right)$, there exists an unique Nash equilibrium, $\mathbf{Q}^{*}(\lambda)$, for the TMGP, and when $\lambda=\lambda^{*}$, the solution is Pareto optimal for the original TMG.

Proof: We rewrite (27) as follows:

$$
I_{i} \frac{\partial v_{i}}{\partial Q_{i}}=W f^{\prime}\left(x_{i}\right)-\frac{\lambda}{\left(1+x_{i} / L_{i}\right)^{2}} .
$$

For $x_{i}$ to be a stationary point, we must have

$$
k_{i}\left(x_{i}\right)=\frac{\lambda}{W}
$$

where

$$
k_{i}\left(x_{i}\right) \equiv\left(1+\frac{x_{i}}{L_{i}}\right)^{2} f^{\prime}\left(x_{i}\right) .
$$

Differentiating $k_{i}\left(x_{i}\right)$, we have

$$
k_{i}^{\prime}\left(x_{i}\right)=\frac{\left(1+x_{i} / L_{i}\right)}{L_{i}}\left[2 f^{\prime}\left(x_{i}\right)+\left(L_{i}+x_{i}\right) f^{\prime \prime}\left(x_{i}\right)\right] .
$$

By (14), we have $k_{i}^{\prime}\left(x_{i}\right)<0 \forall i$, provided that

$$
\min _{i} L_{i} \geq \begin{cases}1.1, & \text { for BIGO } \\ 2.2, & \text { for BSC. }\end{cases}
$$

Note that $k_{i}(0)=f^{\prime}(0)$ and by Property 3 , $\lim _{x_{i} \rightarrow \infty} k_{i}\left(x_{i}\right)=0$. Hence, for any $\lambda \in\left(0, W f^{\prime}(0)\right)$, we can find a unique $x_{i}^{*}$ such that

$$
\frac{\partial v_{i}}{\partial Q_{i}} \begin{cases}>0, & \text { if } 0 \leq x_{i}<x_{i}^{*} \\ =0, & \text { if } x_{i}=x_{i}^{*} \\ <0, & \text { if } x_{i}>x_{i}^{*} .\end{cases}
$$

In other words, $v_{i}$ attains the global maximum at $x_{i}^{*}$. Note that $x_{i}^{*}$ is a strictly decreasing continuous function of $\lambda$. Denote this relation as $x_{i}^{*}(\lambda)$. It is easy to see that $x_{i}^{*} \rightarrow \infty$ when $\lambda \rightarrow 0$, and $x_{i}^{*} \rightarrow 0$ when $\lambda \rightarrow W f^{\prime}(0)$. Thus we can find a unique $\underline{\lambda}$ such that

$$
\sum_{i} \frac{x_{i}^{*}}{x_{i}^{*}+L_{i}}=1
$$

Thus, the inequality

$$
\sum_{i} \frac{x_{i}^{*}}{x_{i}^{*}+L_{i}}<1
$$

holds if $\lambda$ is within the range

$$
\underline{\lambda}<\lambda<W f^{\prime}(0) .
$$

When $\lambda$ decreases from $W f^{\prime}(0), x_{i}^{*}$ increases strictly from zero for all $i$. Consequently, by (20), all the $Q_{i}$ s strictly increase from zero and approach infinity as $\lambda$ tends to $\underline{\lambda}$. Since the power control problem has finite upper bounds, there exists an unique $\lambda^{*}>\underline{\lambda}$ such that $Q_{i}^{*} \leq \tilde{M}_{i}$ for all $i$, and $Q_{j}^{*}=\tilde{M}_{j}$ for some $j$.

Since $Q_{j}^{*}=\tilde{M}_{j}$ for some $j$, by Theorem 1, the solution $\mathbf{Q}^{*}$ is Pareto optimal.

For this theorem to be valid, it requires $L_{i} \geq 2$ for the BIGO model, and $L_{i} \geq 3$ for the BSC model. From now on, we assume that this condition always holds, since this is a mild condition which is satisfied in almost all CDMA systems.

Now we derive a property about the Nash equilibrium. Consider the case $R_{i}>R_{j}$. From (30), it can be seen that

$$
k_{i}(x)>k_{j}(x) \quad \forall x .
$$

From (29), for any given $\lambda$, we have

$$
x_{i}^{*}>x_{j}^{*} .
$$

Hence, we have proven the following.

Theorem 3 (Unequal $E_{b} / J_{0}$ at the Nash equilibrium): If $R_{i}>R_{j}$, then $x_{i}^{*}>x_{j}^{*}$.

We have established a pricing mechanism for the wireless network. The only global information needed is the pricing parameter $\lambda$ and the total received power plus noise. Then the transceiver of each user can be treated as independent entities. Each receiver monitors the throughput and the received power of the corresponding user. The discounted payoff can then be computed and used to drive a close-loop power control algorithm.

\section{Global Property}

We have considered a family of games with pricing parameter $\lambda$. Now we show that playing this family of games is equivalent to solving a family of constrained optimization problems with parameter $\mu$.

The following theorem shows that the Nash equilibrium $\mathbf{Q}^{*}(\lambda)$ has a nice global property.

Theorem 4 (Constrained Global Maximum on Total Throughput): For any $\lambda \in\left[\lambda^{*}, W f^{\prime}(0)\right)$, the Nash equilibrium of the PCGP, $\mathbf{Q}^{*}(\lambda)$, maximizes $C_{T}$, subject to the constraint $\sum_{i} Q_{i}=\mu$ for some $\mu$. Furthermore, $\mu$ is a strictly decreasing function of $\lambda$.

Proof: First consider the constrained optimization problem with parameter $\mu$. With the equality constraint $\sum_{i} Q_{i}=\mu$, there is a one-one monotonic mapping between nonnegative $Q_{i} \mathrm{~s}$ and $x_{i} \mathrm{~s}$

$$
\begin{aligned}
x_{i} & =\frac{Q_{i} L_{i}}{\mu-Q_{i}+N_{0} W}=\frac{Q_{i} L_{i}}{I_{i}} \\
\frac{1}{1+x_{i} / L_{i}} & =1-\frac{Q_{i}}{\mu+N_{0} W} .
\end{aligned}
$$


Thus, it is legitimate to treat $x_{i}$ s as our independent variables. Furthermore, the equality constraint can be rewritten as

$$
\sum_{i=1}^{N} \frac{1}{1+x_{i} / L_{i}}=N-\frac{\mu}{\mu+N_{0} W}
$$

To solve the problem, we make use of the method of Lagrange multiplier. We define the Lagrangian

$$
L=\sum_{i=1}^{N} R_{i} f\left(x_{i}\right)-\tilde{\lambda}\left(\sum_{i=1}^{N} \frac{x_{i}}{x_{i}+L_{i}}-\frac{\mu}{\mu+N_{0} W}\right) .
$$

To maximize $C_{T}$, the following $N+1$ equations must be satisfied:

$$
\begin{aligned}
& \frac{\partial L}{\partial x_{i}}=R_{i} f^{\prime}\left(x_{i}\right)-\frac{\tilde{\lambda} L_{i}}{\left(x_{i}+L_{i}\right)^{2}}=0 \quad \forall i \\
& \frac{\partial L}{\partial \tilde{\lambda}}=\frac{\mu}{\mu+N_{0} W}-\sum_{i=1}^{N} \frac{x_{i}}{x_{i}+L_{i}}=0 .
\end{aligned}
$$

By simple algebraic manipulation, one can show that (43) has the same root as (27) (with $\lambda=\tilde{\lambda}$ ), since

$$
\frac{\partial L}{\partial x_{i}}=\frac{I_{i}}{L_{i}} \frac{\partial v_{i}}{\partial Q_{i}}
$$

Denote the root by $x_{i}^{*}$. Thus, the stationary point, $\mathrm{x}^{*}$, of this optimization problem is just the Nash equilibrium of the TMGP with the Lagrange multiplier, $\tilde{\lambda}$, as the pricing parameter. The nature of this stationary point is governed by the second derivatives

$$
\frac{\partial^{2} L}{\partial x_{i}^{2}}=R_{i} f^{\prime \prime}\left(x_{i}\right)+\frac{2 \tilde{\lambda} L_{i}}{\left(x_{i}+L_{i}\right)^{3}}
$$

and

$$
\frac{\partial^{2} L}{\partial x_{i} \partial x_{j}}=0, \quad i \neq j .
$$

Due to (47), a sufficient condition for the stationary point to be a local maximum becomes

$$
\left.\frac{\partial^{2} L}{\partial x_{i}^{2}}\right|_{\mathbf{x}^{*}}<0 \quad \text { for } i=1,2, \ldots, N .
$$

If we substitute (43) into (46), we have

$$
\begin{aligned}
\left.\frac{\partial^{2} L}{\partial x_{i}^{2}}\right|_{\mathbf{x}^{*}} & =R_{i} f^{\prime \prime}\left(x_{i}^{*}\right)+\frac{2 R_{i}}{L_{i}+x_{i}^{*}} f^{\prime}\left(x_{i}^{*}\right) \\
& =\frac{W L_{i}}{\left(L_{i}+x_{i}^{*}\right)^{2}} k_{i}^{\prime}\left(x_{i}^{*}\right) \\
& <0 .
\end{aligned}
$$

Thus, $\mathbf{x}^{*}$ yields a constrained local maximum.

Since $C_{T}$ is upper bounded by $\sum_{i=1}^{N} R_{i}$, a global maximum exists. Assume that the constrained global maximum of $L$ is attained at $\tilde{\mathbf{x}}=\left(\tilde{x_{1}}, \tilde{x_{2}}, \ldots, \tilde{x_{N}}\right) \neq \mathbf{x}^{*}$. Since the constraint is met, the maximum value of $L$ is independent of the value of $\tilde{\lambda}$. So, we can assume $\tilde{\lambda}$ is chosen to be $\lambda^{*}$ of Theorem 2 . Note that $L$ can be written as

$$
L=\sum_{i=1}^{N} v_{i}\left(x_{i}, \tilde{\lambda}\right)+\frac{\tilde{\lambda} \mu}{\mu+N_{0} W} .
$$

Since $\tilde{\mathbf{x}}$ yields the global maximum, there exists $i$ such that

$$
v_{i}\left(x_{i}^{*}, \tilde{\lambda}\right)<v_{i}\left(\tilde{x}_{i}, \tilde{\lambda}\right)
$$

which violates the fact that the solution is Pareto optimal as guaranteed by Theorem 2 .

Although the solution $\mathrm{Q}^{*}(\lambda)$ maximizes $C_{T}$ on the hyperplane $\sum_{i} Q_{i}=\mu$, it may not be a global maximum over the whole strategy space, $\mathcal{Q}$. Now we assume that the system is interference limited. We make the approximation that $N_{0}=0$. Denote the maximal value of $C_{T}$ over $\mathcal{Q}$ by $C_{\max }$. We have the following result.

Corollary 1: When $N_{0}=0, C_{T}\left(\mathbf{Q}^{*}(\lambda)\right)=C_{\max }$ for any $\lambda \in\left[\lambda^{*}, W f^{\prime}(0)\right)$.

Proof: When $N_{0}=0$, scaling a power vector has no effect on the vector $\mathbf{x}$. Thus, there is no loss of generality to restrict the strategy space into a hyperplane $\sum_{i} Q_{i}=\mu$. Hence, by Theorem $4, Q^{*}$ is optimal.

\section{ASYMPTOTIC ANALYSIS}

In this section, we study a large-scale system in which the number of users, $N$, and the bandwidth, $W$, are both large. We assume that there is no upper bound on the power levels and the transmission rate of each user is bounded as follows:

$$
0<R_{\min } \leq R_{i} \leq R_{\max } \quad \forall i .
$$

Furthermore, we define the spectral efficiency, $\eta$, as the total throughput per unit bandwidth, $C_{T} / W$

$$
\eta=\frac{1}{W} \sum_{i=1}^{N} R_{i} f\left(x_{i}\right) .
$$

We summarize our results in two theorems.

Theorem 5: Let $N=\alpha W$, where $\alpha$ is a constant. There exists $\underline{\lambda}$ such that for any $\lambda \in\left(\underline{\lambda}, W f^{\prime}(0)\right)$, when $W \rightarrow \infty$, we have

$$
x_{i}^{*} \rightarrow \frac{(1-c(\lambda)) W}{\sum_{i=1}^{N} R_{i}} \quad \forall i
$$

where $c(\lambda)$ is a continuous monotonic increasing function of $\lambda$ with

$$
c\left(W f^{\prime}(0)\right)=1
$$

and

$$
c(\underline{\lambda})=0 \text {. }
$$

Proof: Recall that the solution $\mathbf{x}^{*}$ satisfies the following equations $(i=1,2, \ldots, N)$ :

$$
k_{i}\left(x_{i}\right) \equiv\left(1+x_{i} / L_{i}\right)^{2} f^{\prime}\left(x_{i}\right)=\frac{\lambda}{W} .
$$

Occasionally, we use the notation $\mathrm{x}^{*}(\lambda)$ to explicitly show the dependency of $\mathrm{x}^{*}$ on $\lambda$.

Define $\beta$ to be the value such that

$$
f^{\prime}(\beta)=\frac{\lambda}{W} \text {. }
$$

This equation establishes a one-one mapping between $\beta \in[0, \infty)$ and $\lambda \in\left(0, W f^{\prime}(0)\right]$. 
Define $S(\mathbf{x})$ as follows:

$$
S(\mathbf{x}) \equiv \sum_{i=1}^{N} \frac{x_{i} R_{i}}{x_{i} R_{i}+W}
$$

Recall that the feasibility condition of $\mathbf{x}$ requires that

$$
S(\mathbf{x})<1 .
$$

By the property that $k_{i}\left(x_{i}\right)$ is a monotonic decreasing function of $x_{i}$, it can be shown that $x_{i}^{*} \geq \beta$ for all $i$. Thus $S$ is lower bounded as follows:

$$
\begin{aligned}
S & \geq \sum_{i=1}^{N} \frac{1}{1+\frac{W}{\beta R_{\min }}} \\
& =\frac{\alpha}{\frac{1}{W}+\frac{1}{\beta R_{\min }}} .
\end{aligned}
$$

This lower bound is equal to one when

$$
\beta=\frac{1}{R_{\min }}\left[\frac{1}{\alpha-\frac{1}{W}}\right] \text {. }
$$

Define $\beta_{0}$ as follows:

$$
\beta_{0}=\frac{1}{R_{\min }}\left[\frac{1}{\alpha-\frac{1}{W_{0}}}\right]
$$

where $W_{0}$ is an arbitrary constant greater than $1 / \alpha$. Denote the corresponding value of $\lambda$ by $\lambda_{0}$, that is

$$
f^{\prime}\left(\beta_{0}\right)=\frac{\lambda_{0}}{W} \text {. }
$$

When $W>W_{0}$, we have $S\left(\mathbf{x}^{*}\left(\lambda_{0}\right)\right)>1$.

Note that $S(\mathbf{x})$ is a strictly increasing function of each $x_{i}$. Moreover, by (56), all $x_{i}^{*} \mathrm{~s}$ are strictly decreasing function of $\lambda$. Therefore, $S\left(\mathrm{x}^{*}(\lambda)\right)$ is a strictly decreasing function of $\lambda$. When $\lambda=W f^{\prime}(0)$, all $x_{i}^{*}$ s equal zero. Consequently, $S\left(\mathbf{x}^{*}\left(W f^{\prime}(0)\right)\right)=0$.

Hence, for any $W>W_{0}$, there exists a unique $\underline{\lambda}$ (which is greater than $\left.\lambda_{0}\right)$ such that $S\left(\mathbf{x}^{*}(\underline{\lambda})\right)=1$. Therefore, $\mathbf{x}^{*}(\lambda)$ is feasible if $\underline{\lambda}<\lambda<W f^{\prime}(0)$.

For any $\lambda \in\left(\underline{\lambda}, W f^{\prime}(0)\right)$, we have $\lambda / W>\lambda_{0} / W=$ $f^{\prime}\left(\beta_{0}\right)$. Therefore, $\lambda / W$ is lower bounded away from zero. By (56), all $x_{i}^{*}$ s are finite. Hence when $W \rightarrow \infty$, we have $x_{i}^{*} / L_{i} \rightarrow$ 0 . By (56) and the continuity of $f^{\prime}(x)$, we have $x_{i}^{*} \rightarrow \beta$ for all $i$. This proves that all users achieve the same $E_{b} / J_{0}$.

To determine the value of $\beta$, we use the following equality:

$$
S\left(\mathbf{x}^{*}\right)=\sum_{i=1}^{N} \frac{\beta R_{i}}{\beta R_{i}+W}=1-c(\lambda)
$$

where $c(\lambda)$ is a continuous monotonic increasing function of $\lambda$ with $c\left(W f^{\prime}(0)\right)=1$ and $c(\underline{\lambda})=0$.

Since $R_{\min } \leq R_{i} \leq R_{\max }$, we have

$$
\frac{\beta \sum_{i=1}^{N} R_{i}}{W+\beta R_{\min }} \leq 1-c(\lambda) \leq \frac{\beta \sum_{i=1}^{N} R_{i}}{W+\beta R_{\max }}
$$

which can be rearranged as follows:

$$
\frac{(1-c(\lambda)) W}{\sum_{i=1}^{N} R_{i}-(1-c(\lambda)) R_{\min }} \leq \beta \leq \frac{(1-c(\lambda)) W}{\sum_{i=1}^{N} R_{i}-(1-c(\lambda)) R_{\max }} .
$$

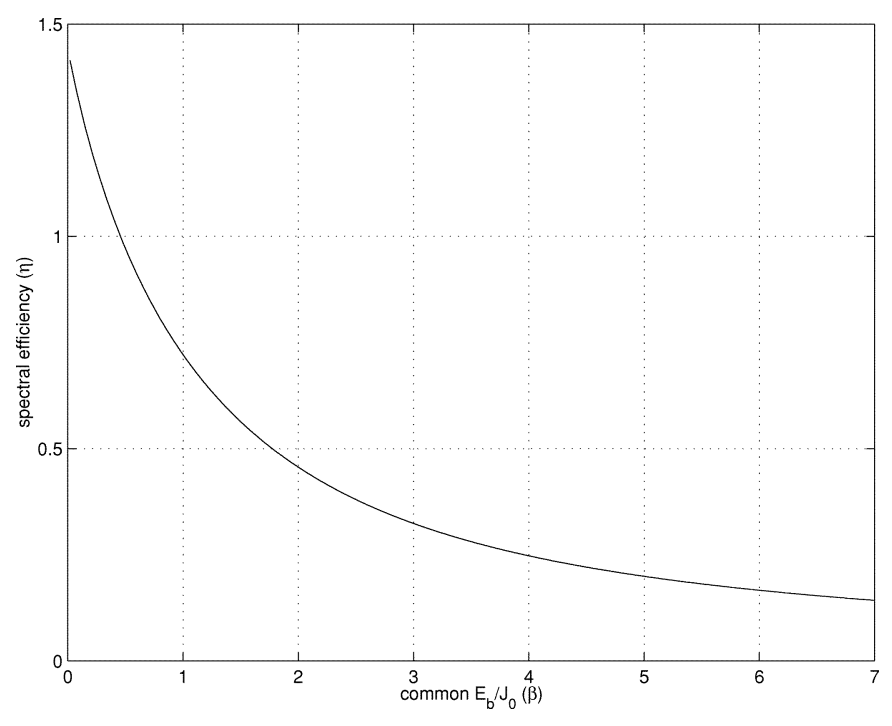

Fig. 3. Spectral efficiency for BIGO channel.

Due to the bounding condition on $R_{i}$, when $N \rightarrow \infty$, we must have $(1-c(\lambda)) R_{\max } / \sum_{i=1}^{N} R_{i} \rightarrow 0$. Hence, we obtain the value of $\beta$

$$
\beta \rightarrow \frac{(1-c(\lambda)) W}{\sum_{i=1}^{N} R_{i}}
$$

Theorem 6: The maximal spectral efficiency $\hat{\eta}$ is given by

$$
\hat{\eta}= \begin{cases}\log _{2} e(\approx 1.44), & \text { for BIGO } \\ \frac{2}{\pi} \log _{2} e(\approx 0.92), & \text { for BSC. }\end{cases}
$$

Proof: We have seen that for large $W, x_{i} \rightarrow \beta=(1-$ $c(\lambda)) W / \sum_{i} R_{i}$ for all $i$. It is easy to see that the supremum of $\beta$ is

$$
\hat{\beta}=\sup _{\lambda \in\left(\underline{\lambda}, W f^{\prime}(0)\right)} \beta=\frac{W}{\sum_{i=1}^{N} R_{i}} .
$$

With this $\hat{\beta}$, the spectral efficiency can be simplified as follows:

$$
\eta=\frac{\sum_{i=1}^{N} R_{i} f(\hat{\beta})}{W}=\frac{1}{\hat{\beta}} f(\hat{\beta}) .
$$

The spectral efficiency for BIGO and BSC are plotted against $\hat{\beta}$ in Figs. 3 and 4, respectively. Note that $\eta$ is a decreasing function of $\hat{\beta}$. It can be shown that the spectral efficiency is maximized when $\hat{\beta} \rightarrow 0$. Thus

$$
\begin{aligned}
\hat{\eta} & =\lim _{\hat{\beta} \rightarrow 0} f^{\prime}(\hat{\beta}) \\
& = \begin{cases}\log _{2} e(\approx 1.44), & \text { for BIGO } \\
\frac{2}{\pi} \log _{2} e(\approx 0.92), & \text { for BSC. }\end{cases}
\end{aligned}
$$

This maximal spectral efficiency is the same as that derived in a single-rate CDMA system [3]. Thus a multirate CDMA 


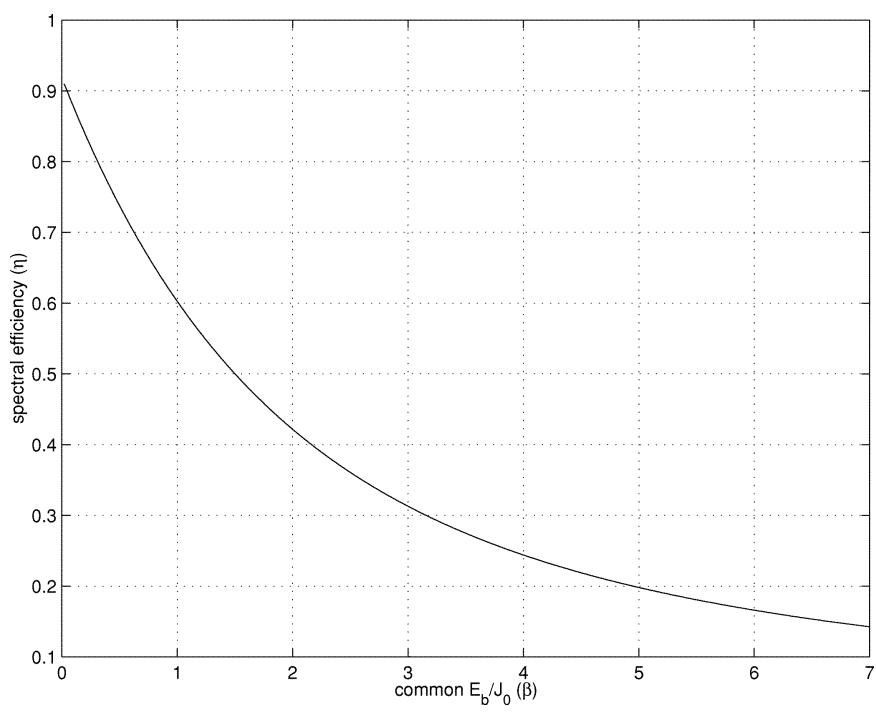

Fig. 4. Spectral efficiency for BSC channel.

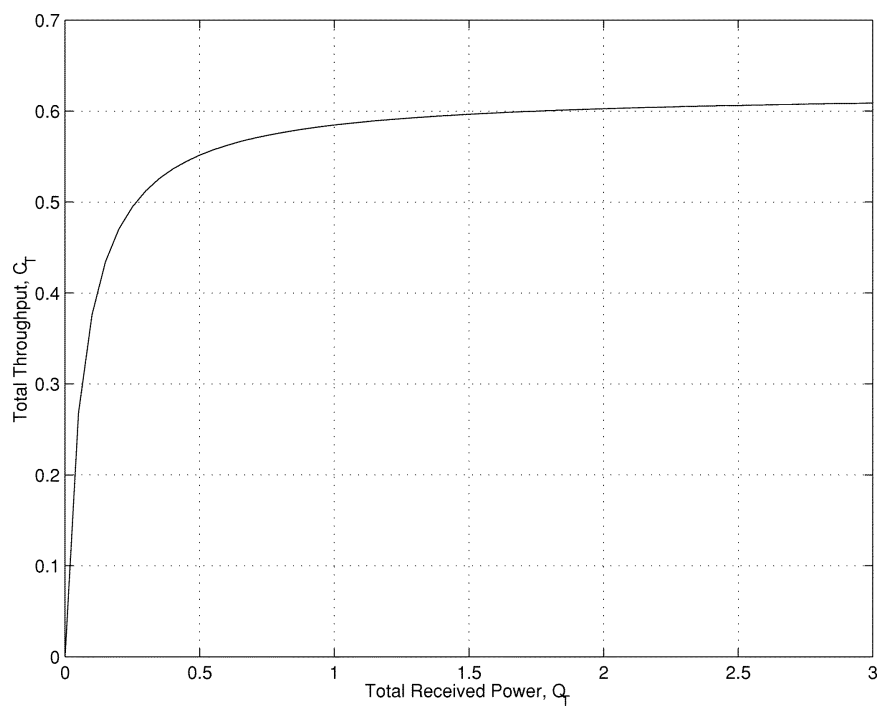

Fig. 5. Tradeoff between the power consumption and the total throughput.

system, with any given rate vector $\mathbf{R}$, can achieve the same spectral efficiency as a single-rate system.

\section{NUMERICAL RESULTS}

In this section, we investigate the effect of the pricing parameter, $\lambda$, on the total throughput, $C_{T}$. For simplicity, we consider a single-rate system. The matched filter output is assumed to be hard quantized such that a BSC model applies. Assume that there are 20 users. Each of them transmits at rate $R=1 \mathrm{Mb} / \mathrm{s}$. The system bandwidth is $20 \mathrm{MHz}$. The noise power is normalized such that unity received power has a signal-to-noise ratio of $10 \mathrm{~dB}$.

As we mentioned before, the effect of $\lambda$ is to constrain the total received power $Q_{T}$. The larger the value of $\lambda$, the smaller the total received power $Q_{T}$. Fig. 5 shows the tradeoff between the power consumption and the total throughput. When $Q_{T} \ll$ 1 , the total throughput grows at a high speed when $Q_{T}$ is increased. This is because the thermal noise dominates in this region, and larger $Q_{T}$ can reduce the effect of noise. When $Q_{T} \gg 1$, the system becomes interference limited. Further increase in $Q_{T}$ has little improvement and the curve levels off.

\section{CONCLUSION}

In this paper, we define a new payoff function for the noncooperative power control game. A new pricing function which improves the system performance is introduced. With this pricing function, the game is shown to possess a unique Nash equilibrium. Furthermore, this equilibrium is shown to maximize the total throughput over a hyperplane with fixed total power. The distance between the hyperplane and the origin can be changed by adjusting a pricing parameter, which is broadcasted by the system.

Moreover, we have studied the system behavior under some asymptotic situation. When both the bandwidth and the number of users are large, the Nash equilibrium can be approximated by keeping the bit energy of all users constant. The resulting spectral efficiencies under the BIGO and the BSC model are derived.

\section{REFERENCES}

[1] T. M. Cover and J. Thomas, Elements of Information Theory. New York: Wiley, 1991.

[2] D. Goodman and N. Mandayam, "Power control for wireless data," IEEE Personal Commun., pp. 48-54, Apr. 2000.

[3] J. Y. Hui, "Throughput analysis for code division multiple accessing of the spread spectrum channel," IEEE J. Select. Areas Commun., vol. 2, pp. 482-486, July 1984.

[4] H. Ji and C. Y. Huang, "Non-cooperative uplink power control in cellular radio systems," Wireless Networks, vol. 4, pp. 233-240, 1998.

[5] K. K. Leung, C. W. Sung, W. S. Wong, and T. M. Lok, "Convergence theorem for a general class of power control algorithms," in IEEE Proc. ICC, Helsinki, Finland, June 2001, pp. 811-815.

[6] T. S. Rappaport, Wireless Communications: Principles and Practice. Englewood Cliffs, NJ: Prentice Hall, 1995.

[7] A. S. Sampath, P. S. Kumar, and J. M. Holtzman, "Power control and resource management for a multimedia CDMA wireless system," in Proc. IEEE PIMRC '95, 1995.

[8] C. U. Saraydar, N. B. Mandayam, and D. J. Goodman, "Pareto efficiency of pricing-based power control in wireless data networks," in Proc. WCNC, 1998.

[9] V. Shah, N. B. Mandayam, and D. J. Goodman, "Power control for wireless data based on utility and pricing," in Proc. IEEE PIMRC, 1998, pp. $1427-1432$.

[10] C. W. Sung, K. K. Leung, and W. S. Wong, "A quality-based fixed-step power control algorithm with adaptive target threshold," IEEE Trans. Veh. Technol., vol. 49, pp. 1430-1439, July 2000.

[11] C. W. Sung and W. S. Wong, "A distributed fixed-step power control algorithm with quantization and active link quality protection," IEEE Trans. Veh. Technol., vol. 48, pp. 553-562, Mar. 1999.

[12] — "Power control and rate management for wireless multimedia CDMA systems," IEEE Trans. Commun., vol. 49, pp. 1215-1226, July 2001.

[13] _ - "Mathematical aspects of the power control problem in mobile communication systems," in Lectures on Systems, Control, and Information: Lectures at the Morningside Center of Mathematics, L. Guo and S. S.-T. Yau, Eds: International Press, 2000.

[14] S. Verdù and S. Shamai, "Spectral efficiency of CDMA with random spreading," IEEE Trans. Inform. Theory, vol. 45, Mar. 1999.

[15] A. J. Viterbi and J. K. Omura, Principles of Digital Communication and Coding. New York: McGraw-Hill, 1979.

[16] R. D. Yates, "A framework for uplink power control in cellular radio systems," IEEE J. Select. Areas Commun., vol. 13, pp. 1341-1347, Sept. 1995.

[17] J. Zander, "Performance of optimum transmitter power control in cellular radio systems," IEEE Trans. Veh. Technol., vol. 41, pp. 57-62, Feb. 1992. 


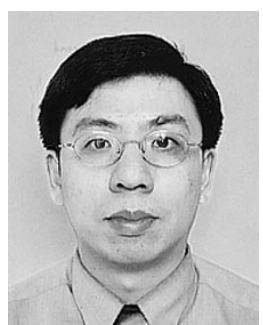

Chi Wan Sung (M'98) received the B.Eng., M.Phil., and $\mathrm{Ph} . \mathrm{D}$. degrees in information engineering from the Chinese University of Hong Kong, in 1993, 1995, and 1998, respectively.

After graduation, he was appointed Visiting Assistant Professor with the Department of Information Engineering, Chinese University of Hong Kong. He joined the City University of Hong Kong in 2000 and was an Assistant Professor in the Department of Computer Science. Since September 2001, he has been with the Department of Computer Engineering and Information Technology. His research interests include the area of wireless networks with an emphasis on power control, resource management, and signature sequence adaptation for CDMA systems.

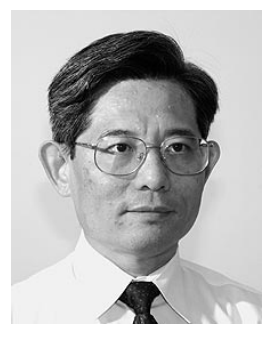

Wing Shing Wong (M'81-SM'90-F'02) received the combined M.A.B.A. degree in 1976 (summa cum laude), from Yale University. He received the M.S. degree in 1978 and the Ph.D. degree in 1980, both from Harvard University, Cambridge, MA.

He joined AT\&T Bell Laboratories in 1982. From 1987 to 1992 , he managed a group of technical staff working on a number of research and development projects and consulting activities. He joined the Chinese University of Hong Kong in 1992 and is now a Professor of Information Engineering. He has been the Chairman of the Information Engineering Department since 1995. He is actively involved in a variety of $R \& D$ projects including topics such as mobile communication systems, search engine development, and information issues in estimation and control. He has published over 90 refereed journal and conference papers and is the recipient of multiple competitive R\&D grants from the Hong Kong Research Grant Council and the Industry Support Fund. He leads a project on a Chinese search engine, MoLi \& ANSeRS, which has been licensed to several high-tech companies. He is a Visiting Professor of Southeast University, Nanjing.

Dr. Wong was an Associate Editor of the IEEE TRANSACTIONS ON Automatic ConTROL for four years. He is the co-Editor-in-Chief of a new journal, Communications in Information and Systems. He is a Member of the 7th Council of the Chinese Association of Automation. 